\title{
Perceptions of undergraduate dental students at Makerere College of Health Sciences, Kampala, Uganda towards patient record keeping
}

\author{
A M Kutesa, ${ }^{1}$ BDS, MSc (Dent); J Frantz, ${ }^{2} \mathrm{PhD}$ \\ ${ }^{1}$ Department of Dentistry, College of Health Sciences, Makerere University, Kampala, Uganda \\ ${ }^{2}$ Department of Physiotherapy, Faculty of Community and Health Sciences, University of the Western Cape, Cape Town, South Africa
}

Corresponding author: A M Kutesa (akutesa@chs.mak.ac.ug)

Background. The creating, maintenance and storage of patients' medical records is an important competence for the professional training of a dental student. Objective. Owing to the unsatisfactory state of dental records at the students' clinic, the objective of this study was to obtain information from undergraduate dental students on the factors that affect this process and elicit recommendations for improvement.

Methods. This qualitative cross-sectional study used focus group discussions with 4th- and 5th-year dental students for data collection. Data were captured through a written transcript and an audio recorder. The data were transcribed and analysed manually through developing themes, which were compared with the literature and interpreted.

Results. Three themes emerged: (i) Poorly designed clerking forms. The clerking forms were deemed to have a poor design with inadequate space for clinical notes. It was recommended that they be redesigned. (ii) Inadequate storage space. Space for storing patient records was deemed inadequate and a referencing system for file retrieval was lacking. It was recommended that more space be allocated for storage, with a referencing system for easy file retrieval. (iii) Poor maintenance of records. Patients' records, especially radiographs, were not well labelled and stored. It was recommended that drug envelopes be utilised to store radiographs. An electronic system was deemed the ultimate solution to this problem.

Conclusion. The general perception was that the current paper-based record system at the clinic was unsatisfactory. Therefore, there is a need to improve the maintenance and storage of records, and to change to a more efficient electronic system. The students' attitude towards record keeping was found to be questionable, with a need to be addressed as part of teaching and learning in the curriculum. Lecturers were deemed to have a bigger role to play in the record-keeping process.

Afr J Health Professions Educ 2016;8(1):33-36. DOI:10.7196/AJHPE.2016.v8i1.521

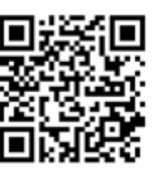

The creating of clear and accurate patient medical records, and their maintenance and storage, are an important part of the professional training of a dental student. The records of an individual patient in a dental setting constitute the clinical notes, radiographs and plaster models, as well as other information. These records present an in-depth and continuous documentation of the patient's condition, which helps to contribute to both diagnosis and proper patient care. ${ }^{[1,2]}$ In addition, these records have various other purposes, including research, administration, quality assurance, teaching and learning, and can be used as evidence during legal proceedings. ${ }^{[3,4]}$

Patient records have traditionally been paper-based but electronic documentation, in addition to residual paper-based records, is being increasingly used because of the inherent disadvantages of paper-based systems. Documented problems of paper-based systems in the literature ${ }^{[5]}$ include difficulty in deciphering clinician handwriting, difficulty in accessing patient information from remote centres, time-consuming patient chart retrieval, and questionable security and confidentiality of records. ${ }^{[6]}$

The other challenge identified with paper-based records is their incompleteness, ${ }^{[6,7]}$ i.e. the omission of vital information, such as laboratory results or radiography reports, from the patient record. Missing information has been shown to have consequences, including delays in patient care, repeated laboratory testing and additional patient visits, which also adversely affect the wellbeing of patients. In addition to the pressure to reduce patient length of stay in hospital, this highlights the need for investigation into the use of electronic records. The pace of modern practice dictates the use of technology, but the lack of direct clinical input in the development of information technology is a major factor in the failure of many clinical information systems ${ }^{[8]}$ Replacing paper records with electronic records will assist in changing medical practice, including relationships between doctors and nurses, responsibilities, and work distribution. However, clinicians need to involve themselves in ensuring that software for documenting patient encounters complements the way they work.

Documentation and note taking continue to be an area of concern because quality documentation is an important aspect of communication by health professionals. The notes of any health professional serve as a window into their thought processes. ${ }^{[9]}$ During student training, communication through note taking is an important aspect of exchange of information with colleagues and patients. Therefore, teaching and learning of students in written and verbal communication skills is a key component of health professional curricula. Some academics ${ }^{[10]}$ have advocated that it is now more important than ever that education and practice in professional writing and speaking should be included as part of medical training and residency. Furthermore, national quality-regulating bodies such as the Accreditation Council for Graduate Medical Education in the USA, a body that establishes educational standards, have included interpersonal skills and communication as one of six core competencies for all training programmes. ${ }^{[11]}$

At the Makerere College of Health Sciences (MakCHS), Kampala, Uganda, teaching and learning in interpersonal and communication skills are included in the undergraduate dental curriculum. These skills are expected to be 
demonstrated by the students, particularly during the clinical years, when they interact with other professionals and patients through record keeping. At MakCHS dental clinic, the record-keeping system is paper based and patient records have recently had many challenges, which include the loss of patient records, poor status and incomplete records. Moreover, the storage of the patient records is inadequate, using cardboard boxes as a filing system, making it difficult to trace patient information when they come for recall visits. These challenges have had an effect on student learning and patient care. Despite these concerns, no study has been done among the faculty and students to document their perceptions of the status quo.

The objective of this study was to document the perceptions of undergraduate dental students at MakCHS of factors that affect the keeping of clear and accurate patient records, and their maintenance and storage. It also elicits recommendations to improve the system for better teaching and learning at MakCHS.

\section{Methods}

\section{Research setting and design}

This was a cross-sectional study using descriptive qualitative methodology, conducted at the Department of Dentistry, MakCHS.

\section{Population and sampling}

The study population comprised 4th- and 5th-year dental students $(n=20)$ and faculty members $(n=2)$. Fourth- and 5 th-year students were selected to participate because, at this time, they were in their clinical clerkships and actively used medical records as part of patient care. Of the 18 faculty members at the Department of Dentistry, two were purposefully selected to participate in the study, as they were actively involved in student training for this competence and supervision at the clinic.

\section{Data collection methods}

The instrument used for data collection comprised a structured focus group discussion exploring the creating, maintenance and storage of patient records. The interview guide was developed by the authors to ensure that participants were allowed to discuss their perceptions concerning different aspects of patient record-keeping competence, which included knowledge on patient charting, its existence in the curriculum, gaps identified, and recommendations to improve the curriculum.

Two focus group discussions were conducted. One group comprised 4th-year dental students and the other 5th-year dental students. The dental classes are small, with 10 - 15 students per year, and thus all students were invited to participate in the study. A total of 10 students per focus group were interviewed, and the discussions took place in a lecture room at the Department of Dentistry for an hour each. Focus group discussions were conducted by one of the researchers (AK) using a structured interview guide. A 5th-year dental student was used as a research assistant and captured the discussions through a written script and a voice recorder.

To establish the trustworthiness of this research, the concepts of credibility, transferability, dependability and confirmability were considered as decisive factors for quality, based on Guba's four criteria for trustworthiness. ${ }^{[12]}$ Credibility was addressed by describing the methods in detail in the protocol to convey the actual situations that would be investigated. To allow transferability, sufficient detail of the study context has been reported in this article to enable other researchers to decide whether the setting is similar to others and whether the findings can be applied to other settings. Dependability has been achieved by describing the methodology clearly to enable a future investigator to repeat the study. To achieve confirmability, the findings that emerged from the data were supported by quoting the participants' words during the discussions.

\section{Data analysis}

After the interviews, the data were transcribed and checked for correctness by cross-checking with the three data sources (the notes of the researcher, the research assistant and the recorder). Data were coded by the author using the open code system. ${ }^{[13]}$ Coding was done by first using a start list of preset codes. The initial preset codes were three: making, maintenance and storage, which were derived from the study objective. Themes were developed from the coded data with the help of an expert in qualitative analysis, and the emerging themes were compared with the data and finally interpreted. The participants' quotes were identified and labelled using a method devised by the authors. As an example, a quote would be labelled with the year of study as the first digit and the participant number as the second digit. Y3P1 would signify a 3rd-year student, participant 1 . The emerging themes were checked by one of the authors (JF).

\section{Ethical considerations}

Ethical clearance to carry out the study was obtained from the School of Medicine Review and Ethics Committee. Prior to commencement, gatekeeper permission was obtained from the Department of Dentistry and the Faculty. Written consent was obtained from the selected participants in accordance with the Helsinki Declaration. The investigators informed the participants of the procedure, its objectives and benefits, and any possible risks involved. Confidentiality was ensured by avoiding the use of any person identifiers.

\section{Results}

The two focus group discussions consisted of a total of 20 participants, of whom 10 were female and 10 were male. The 5 th-year group had 7 females and 3 males, and the 4th-year group had 4 females and 6 males.

The transcribed data from the interviews produced three themes: poorly designed clerking forms, inadequate storage space and poor maintenance of records. The following issues within the themes were highlighted: inadequacy of the currently used clerking forms, patient files, labelling and the electronic system.

\section{Poorly designed clerking forms}

It emerged that the current clerking forms were in need of a redesign as they have insufficient space to make clinical notes, and seemed disorganised in design. In addition, owing to the texture of the paper used, they were not easy to store.

'Clerk forms do not have enough space for patient recall notes.' (Y5P2)

'The clerk form is disorganised, they need to be re-designed.' (Y5P1)

'Record keeping should be improved.' (Y4P6)

\section{Inadequate storage space}

The participants felt that the current space for storing patient records was inadequate. In addition, referencing to facilitate patient file retrieval needed updating. It was recommended that management use box files to store 
patient records and provide a bigger storage area with a reference system for ease of file retrieval.

'We need more room to store the patient records, our lockers are too small.' (Y5P3)

'We need a reference system to be able to retrieve old patient records'. (Y4P9)

'We don't know how to get the records, apart from using patient names. (Y4P9)

'Sometimes we end up reclerking or asking the patient their previous treatment.' (Y4P9)

\section{Maintenance}

It emerged that patient records, especially radiographs, were not well labelled. Furthermore, their storage was poor. It was unanimously recommended that drug-dispensing envelopes should be labelled and used to store patient radiographs; the introduction of an electronic system to improve storage was also recommended.

'X-rays should be kept in labelled envelopes and stored in files.' (Y5P6)

'We need the electronic system for better storage.' (Y5P7)

The consensus was that there was an urgent need to introduce an electronic system for better record keeping. Respondents also felt that the lecturers had a bigger role to play in record keeping but had not emphasised the aspects of maintenance and storage of patient records. The students also felt that they needed more training in record keeping and guidance from the supervisors.

'All lecturers at the clinic should emphasise record keeping.' (Y5P1)

'Supervisors at the clinic do not put in enough.' (Y4P2)

The recommendations that were given were practical and easy to implement. The students also thought that the responsibility of good record keeping lay with their supervisors and not with themselves.

'X-rays should be kept in labelled envelopes and stored in files.' (Y5P6)

'Provide box files for each of us to keep the patient records.' (Y4P2)

'Someone should be hired for record keeping.' (Y4P2)

'Lecturers have a bigger role to play in record keeping but they have not put a lot of emphasis on it.' (Y4P1)

\section{Discussion}

Record keeping is an important competence that, as a key responsibility of the dentist in clinical practice, must be developed during the professional training of a dental student. A dentist is obliged, ethically and legally, to keep patient records that are accurate, legible, comprehensive and organised because appropriate decisions are based on accurate and complete facts retrievable from a patient's record. ${ }^{[14]}$ This is emphasised through various guidelines from medical and dental councils internationally, ${ }^{[15-17]}$ which state that patient records must be clear and accessible. According to these guidelines, records must formally record patient details, clinical findings and treatment given, among other details. These records should be kept securely and in line with dataprotection requirements of the institution for a period of not less than 10 years from when the patient last attended the clinic for treatment. ${ }^{[17]}$

Owing to the inadequacies observed by the authors with patient records at MakCHS dental school clinic, the next step was to obtain information and create awareness among the undergraduate dental students and the faculty of the factors that affect this process, with the overall objective of improving the quality of service rendered by dental professionals.

In view of the international guidelines discussed above, ${ }^{[15,16]}$ we found that the students felt that the dental records were being inadequately stored and maintained at the clinic. We thought this was unfortunate because accurate records, in part, are considered important for good clinical decisions ${ }^{[13]}$ and, therefore, better treatment outcomes.

The students expressed the need for more training in record keeping and guidance from the supervisors. This information was beneficial to educators and will guide us in improving student training at the clinic, especially supervision. This finding is corroborated elsewhere, where students have been found to be a source of information that can provide formative feedback to faculty for improving teaching, course content and structure. ${ }^{[18]}$ We also found that the students were able to give recommendations that are practical and easy to implement. As an example, they suggested the use of drug envelopes and box files to store patient radiographs and records. These recommendations we believe will be taken into consideration as we plan to improve patient record storage and maintenance.

The reaction of the students in this study was interesting, showing that they expected the challenges to be solved by someone else. They felt that the responsibility for good record keeping lay entirely with others rather than with the students. They commented that someone should be hired for record keeping and that lecturers had a bigger role to play in record keeping. This showed us that the students were aware of inadequate recordkeeping practices at the clinic, but that they took no initiative to effect change to improve the situation. This implies that the students cannot easily show initiative to change challenging situations, even when they are aware of them, expecting others to take the lead. Therefore, we realise that students need to be taught to be agents of change, and advocate change in their work environment to improve practice. ${ }^{[19]}$ This remains a challenge, as the problem will not be solved if students do not accept this responsibility during their training. Educators will need to ensure that this aspect in the curriculum is highlighted and receives the attention it deserves.

\section{Conclusion}

The general perception was that the current paper-based record system at the clinic was unsatisfactory. Therefore, there is a need to improve the storage of records and their maintenance, and to change to a more efficient electronic system. The students' attitude to record keeping was also found to be questionable, emphasising a need to be addressed as part of teaching and learning in the curriculum. Supervisors need to take on a more supportive role and guide the students towards good record-keeping practices. Medical record-keeping clinical skills are a core part of the training of a dental student and should be given the platform they deserve in the curriculum.

Acknowledgements. We highly appreciate the support of both the Medical Educational Partnership Initiative/Medical Education for Equitable Services for All Ugandans (MEPI/MESAU) and the Sub-Saharan Africa-FAIMER Regional Institute (SAFRI) accorded during the course of this study. 


\section{References}

1. Cole A, McMichael A. Audit of dental practice record-keeping: A PCT-coordinated clinical audit by Worcestershire dentists. Prim Dent Care 2009;16(3):85-93. [http://dx.doi.org/10.1308/135576109788634296] 2. Osborn JB, Stoltenberg JL, Newell KJ, Osborn SC. Adequacy of dental records in clinical practice: A survey of dentists. J Dent Hyg 2000;74(4):297-306.

3. Charangowda BK. Dental records: An overview. J Forensic Dent Sci 2010;2(1):5-10. [http://dx.doi. org/10.4103/0974-2948.71050]

4. Soisson EL, van de Creek L, Knapp S. Thorough record keeping: A good defense in a litigious era. Professional Psychology: Research and Practice 1987;18(5):498.

5. Zandieh SO, Yoon-Flannery K, Kuperman GJ, Langsam DJ, Hyman D, Kaushal R. Challenges to EHR implementation in electronic versus paper-based office practices. J Gen Intern Med 2008;23(6):755-761. [http:// dx.doi.org/10.1007/s11606-008-0573-5

6. Hersh WR. The electronic medical record: Promises and problems. J Am Soc Inf Sci 1995;46(10):772-776

7. Smith PC, Araya-Guerra R, Bublitz C, et al. Missing clinical information during primary care visits. J Am Med Ass 2005;293(5):565-571. [http://dx.doi.org/10.1001/jama.293.5.565]

8. Walsh S. The clinician's perspective on electronic health records and how they can affect patient care. Br Med 2004;328(7449):1184-1187. [http://dx.doi.org/10.1136/bmj.328.7449.1184]

9. Cegala DJ, Broz SL. Physician communication skills training: A review of theoretical backgrounds, objectives and skills. Med Educ 2002;36(11):1004-1016.
10. Simonson JA. Why we must teach written and verbal communication skills to medical students and residents. Acad Med 2013;88(4):435. [http://dx.doi.org/10.1097/ACM.0b013e3182854f57]

11. Accreditation Council for Graduate Medical Education (ACGME). Core competences. 2003. http://www.ecfmg org/echo/acgme-core-competencies.html (accessed 14 July 2014).

12. Guba EG. Criteria for assessing the trustworthiness of naturalistic inquiries. Educ Commun Tech 1981;29(2):75-91.

13. Lofland J, Lofland LH. Analyzing Social Settings: A Guide to Qualitative Observation and Analysis. Belmont, USA: Wadsworth Publishing Company, 1995.

14. Record Keeping Outline - Oregon.gov. www.oregon.gov/OBCE/publications/record_keeping_ch_final_jan06. pdf (accessed 14 July 2014)

15. The Guidelines of the Royal College of Dental Surgeons, May 2008. www.rcdso.org (accessed 8 July 2014).

16. Good medical practice. 2013. http://www.gmc-uk.org/guidance/ (accessed 14 July 2014).

17. Record keeping in the UK. 2012. http://www.dentalprotection.org/UK/RiskManagement/RecordKeeping/ (accessed 8 July 2014)

18. Marsh HW, Roche L. The use of students' evaluations and an individually structured intervention to enhance university teaching effectiveness. Am Educ Res [ 1993:30(1):217-251. [http.//dx doiorg/103102/00028312030001217] 19. Dunne E Roos Z Tony Brown T Nurser T. Students as change agents: New ways of engaging with learning and teaching in higher education. York, UK: ESCalate/Higher Education Academy Publication, 2011. 